\title{
Optical sensor based on hybrid LPG/FBG in D-fiber for simultaneous refractive index and temperature measurement
}

\author{
Xianfeng Chen*, Kaiming Zhou, Lin Zhang, Ian Bennion \\ Photonics Research Group, Aston University, Birmingham, B4 7ET, U.K. \\ Tongyu Liu \\ Intelligent Sensor Systems Ltd, Brunel Science Park, Uxbridge, Middx UB8 3PQ, U.K.
}

\begin{abstract}
A dual-parameter optical sensor has been realized by UV-writing a long-period and a Bragg grating structure in D-fiber. The hybrid configuration permits the detection of the temperature from the latter and measuring the external refractive index from the former responses, respectively. The employment of the D-fiber allows as effective modification and enhancement of the device sensitivity by cladding etching. The grating sensor has been used to measure the concentrations of aqueous sugar solutions, demonstrating the potential capability to detect concentration changes as small as $0.01 \%$.
\end{abstract}

Keywords: Optical sensor, D-fiber, etching, hybrid LPG/FBG, external refractive index, temperature

\section{INTRODUCTION}

Optical fiber sensors based on fiber Bragg grating (FBG) and long-period grating (LPG) structures have attracted considerable interests for applications, including monitoring temperature, strain, loading, bending, pressure, vibration of many engineering systems and structures $[1,2]$. The light coupling takes place between the forward- and backwardpropagating core modes in an FBG and between the forward-propagating core and cladding modes in an LPG. It is the involvement of the cladding mode coupling in the latter making the LPG structure intrinsically sensitive to external refractive index (ERI) and this unique ERI sensitivity has been utilized for implementation of optical chemsensors [3-6].

Due to the large inherent thermal and ERI cross-sensitivity, conventional LPGs are not ideal chemsensor devices for ERI encoded chemical concentration measurement. To date, few reports have been demonstrated to overcome the thermal and ERI cross-sensitivity [4, 5]. In this paper, we propose a hybrid LPG/FBG structure UV-inscribed in a D-fiber for simultaneous measurement of temperature and ERI. The etched hybrid gratings with remarkably enhanced ERI sensitivity have been used to measure concentrations of aqueous sugar solutions.

\section{DEPENDENCE OF TEMPERATURE AND EXTERNAL REFRACTIVE INDEX}

The mode coupling in an FBG and LPG can occur only at phase match wavelengths

$$
\begin{aligned}
& \lambda_{B}=2 n_{c o}^{e f f} \Lambda_{B} \\
& \lambda_{L P}=\left(n_{c o}^{e f f}-n_{c l, m}^{e f f}\right) \Lambda_{L P}
\end{aligned}
$$

* chenx2@aston.ac.uk; phone 44-121-3593611 ext 4969 
where $n_{c o}^{e f f}$ and $n_{c l, m}^{e f f}$ are the effective indexes of the core mode and the $m t h$ cladding mode, respectively, and $\Lambda_{B}$ and $\Lambda_{L P}$ are the periods of FBG and LPG, respectively. For a hybrid LPG/FBG structure, we may measure the resonance responses of FBG and LPG and construct a matrix as

$$
\left(\begin{array}{l}
\Delta \lambda_{B} \\
\Delta \lambda_{L P}
\end{array}\right)=\left[\begin{array}{ll}
A & 0 \\
C & D
\end{array}\right]\left(\begin{array}{l}
\Delta T \\
\Delta n_{e x}
\end{array}\right),
$$

where $\Delta T$ and $\Delta n_{e x}$ are thermal and ERI perturbation, respectively. A is the temperature coefficient of FBG, C and D are the temperature coefficient for LPG responses, respectively. The three coefficients A, C and D can be defined by measuring separately the temperature and ERI responses of FBG and LPG of the hybrid structure. Therefore one can determine the two measurands, $\Delta T$ and $\Delta n_{e x}$, simultaneously by a decoupling signal processing employing the inverse matrix

$$
\left(\begin{array}{l}
\Delta T \\
\Delta n_{e x}
\end{array}\right)=\left[\begin{array}{ll}
A & 0 \\
C & D
\end{array}\right]^{-1}\left(\begin{array}{l}
\Delta \lambda_{B} \\
\Delta \lambda_{L P}
\end{array}\right)
$$

\section{EXPERIMENT}

\subsection{Fabrication of hybird LPG/FBG in D-fiber}

The hybrid gratings used in our experiments were fabricated in the hydrogenated D-fiber by exposing the fiber to $244 \mathrm{~nm}$ UV laser. The $40 \mathrm{~mm}$-long LPG of $350 \mu \mathrm{m}$ period was first inscribed in the fiber employing the point-by-point technique and the FBG of $3 \mathrm{~mm}$ length was then written adjacent to the LPG using the phase mask method (Fig. 1a). After the UV exposure, the hybrid gratings were annealed at $85^{\circ} \mathrm{C}$ for $24 \mathrm{~h}$ to stabilize their optical properties. The transmission spectra plotted in Fig.1b were measured using a broadband light source, a polarization controller and an optical spectrum analyzer. There are two sets of broad LPG resonances, corresponding to the two orthogonally polarized modes $\left(\mathrm{P}_{1}\right.$ and $\mathrm{P}_{2}$ ), caused by the birefringence of D-fiber with asymmetrical cladding and the elliptical core.

(a)

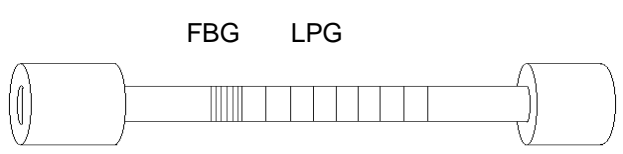

(b)

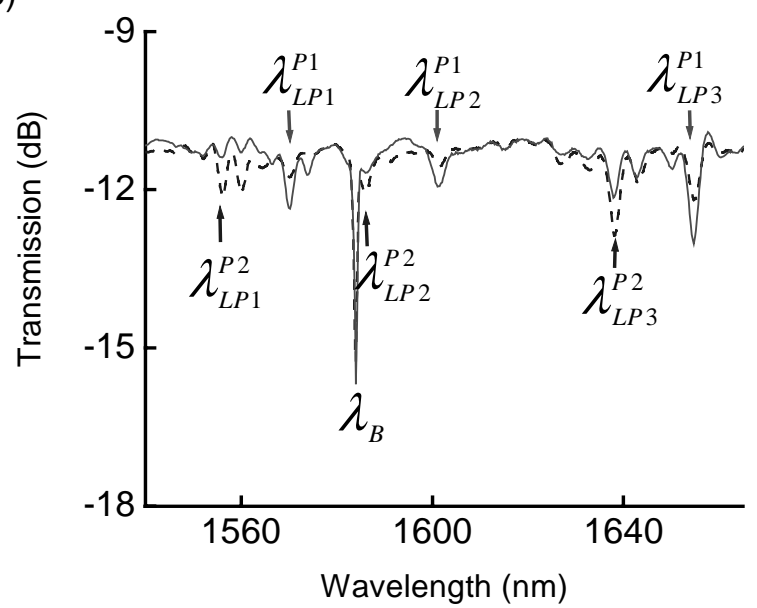

Fig.1 (a) Schematic diagram of a hybrid LPG/FBG structure; (b) typical transmission spectra of a hybrid LPG/FBG in D-fiber. 


\subsection{HF etching process}

The hybrid LPG/FBG were immersed in the $10 \%$ concentration hydrofluoric acid (HF) solution. It was noticed from Fig.2 that, with increasing etching time, the FBG response remained intact while all attenuation bands of LPG shifted toward longer wavelengths and the extent of the red-shift increased with mode order. After etching 50min, the red shift of $\lambda_{L P 1}^{P 1}, \lambda_{L P 2}^{P 1}$ and $\lambda_{L P 3}^{P 1}$ are $40.5 \mathrm{~nm}, 61 \mathrm{~nm}, 78 \mathrm{~nm}$, respectively.

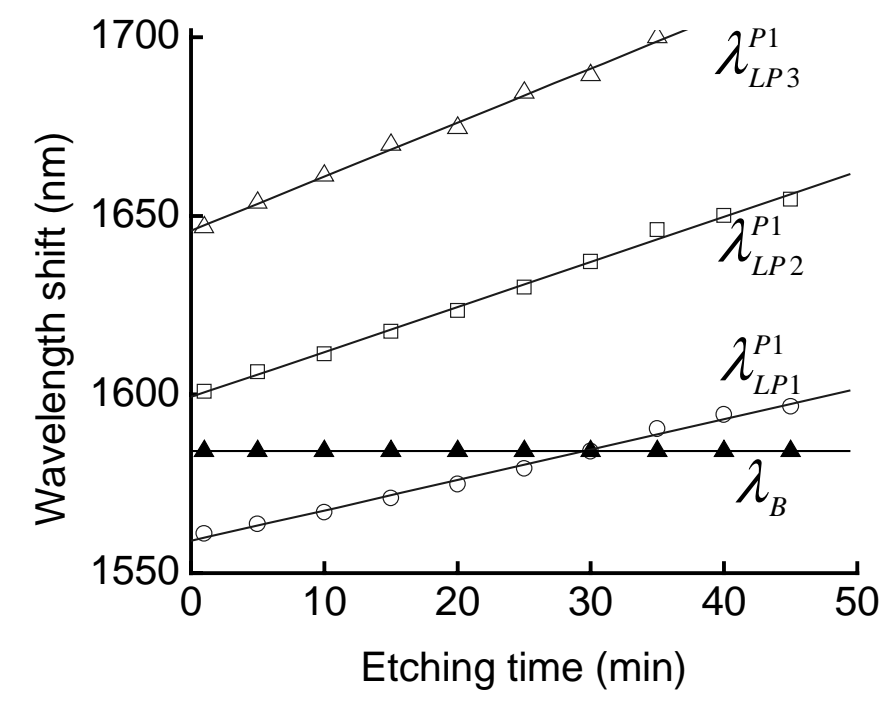

Fig.2 HF etching-induced wavelength shifts of three resonances of the LPG (open symbols) and FBG (solid symbol).

\subsection{Temperature characteristics}

In order to make a comparative study, both etched and unetched gratings fabricated at the same condition were investigated. The temperature sensitivity was characterized by heating the gratings from $10^{\circ} \mathrm{C}$ to $60^{\circ} \mathrm{C}$ using a peltier with a temperature control accuracy of $0.1^{\circ} \mathrm{C}$. To minimize the thermal and bend cross-sensitivity in the latter experiment, the gratings were placed in a straight $\mathrm{V}$-groove on a metal plate and immersed in the sugar solution and kept at a constant temperature.

Fig. 3a depicts the transmission spectra of the LPG resonance $\lambda_{L P 1}^{P 1}$ and the FBG resonance $\lambda_{B}$ of the etched hybrid grating at $10^{\circ} \mathrm{C}$ and $60^{\circ} \mathrm{C}$. Fig $3 \mathrm{~b}$ plots the wavelength shift against temperature for $\lambda_{L P 1}^{P 1}$ and $\lambda_{B}$ of the etched and unetched gratings. Clearly, $\lambda_{L P 1}^{P 1}$ and $\lambda_{B}$ of both gratings shift linearly toward longer wavelengths when the temperature was increasing. It can be noted from Fig. $3 b$ that there is no significant change in FBG thermal response after etching, but the temperature sensitivity of LPG has been reduced dramatically for the etched structure. The linear thermal responses of LPG and FBG permit us to calibrate the temperature coefficients A and C of the sensitivity matrix. From Fig 3b, we estimate that $A=11.3 \mathrm{pm} /{ }^{\circ} \mathrm{C}$ are the same and $C=96.8 \mathrm{pm} /{ }^{\circ} \mathrm{C}$ and $48.1 \mathrm{pm} /{ }^{\circ} \mathrm{C}$ for unetched and etched gratings, respectively. We see that the thermal sensitivity of LPG is halved after the etching, but it is still more 
than four times of that of FBG, which is still significant enough to impose a temperature cross-sensitivity on the LPG response.
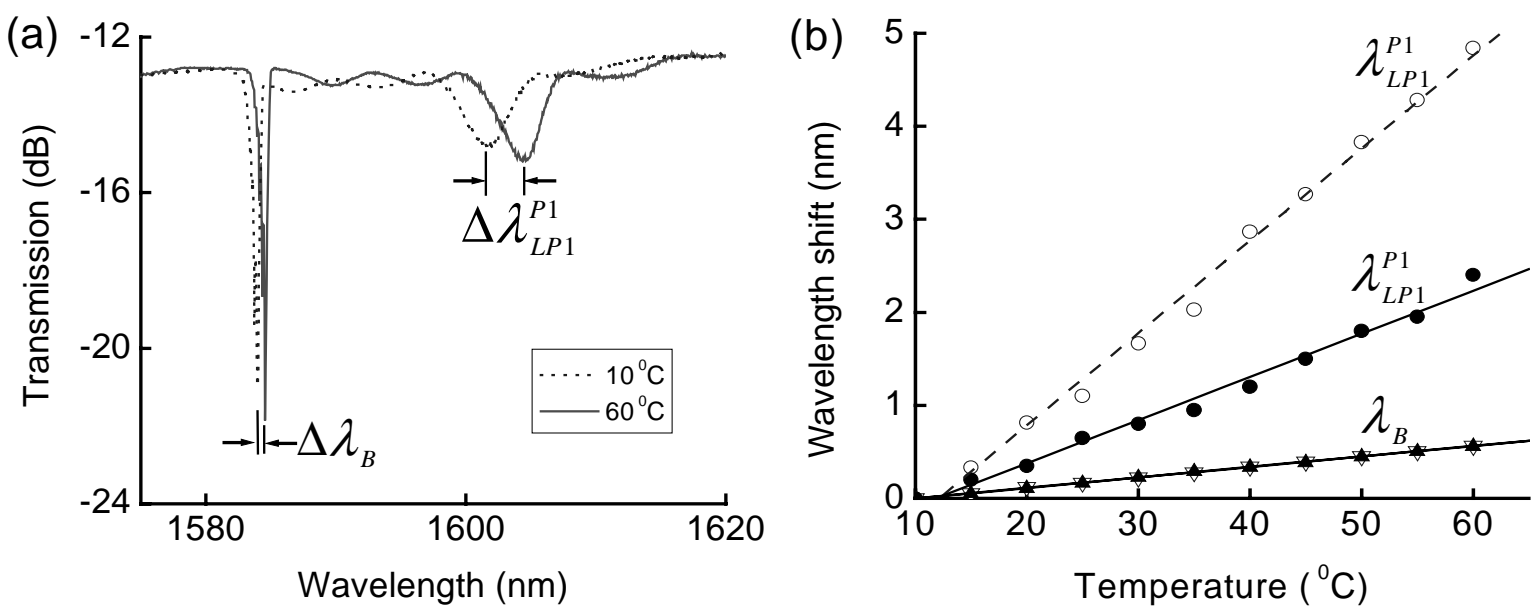

Fig. 3 (a) Transmission spectra of the etched LPG/FBG measured at $10^{\circ} \mathrm{C}$ and $60^{\circ} \mathrm{C}$. (b) The wavelength shift against temperature for unetched (open symbols) and etched (solid symbols) LPG/FBG devices.

\subsection{Sugar concentration measurement and sensitivity characterization}

We have evaluated the ERI sensitivities of etched and unetched hybrid LPG/FBG devices by subjecting them in a set of aqueous sugar solutions with concentration ranging in 0\%-60\%. For sugar concentration change from $0 \%$ to $60 \%$, the refractive index increases more or less linearly from 1.33 to 1.44 [7]. Fig. 4a shows the spectral evolution of the etched hybrid LPG/FBG sensor under a series of sugar solutions. Fig. 4b plots the ERI-induced wavelength shifts of FBG ( $\lambda_{B}$ ) and LPG $\left(\lambda_{L P 1}^{P 1}, \lambda_{L P 2}^{P 1}\right)$ for etched and unetched gratings. Note, the third cladding mode $\left(\lambda_{L P 3}^{P 1}\right)$ was moved out from the measurement range. Evidently, the FBG response is totally insensitive to the ERI whereas the LPG resonances shift nonlinearly toward shorter wavelengths with increasing ERI. Furthermore, we see two clear trends from Fig. 4b: (1) higher order mode has a higher ERI sensitivity; (2) etching has enhanced the ERI sensitivity, especially more effective in 1.42-1.44 range. We may select two near-linear ERI response ranges: $1.33-1.40$ and 1.42-1.44. If one defines ERI sensitivity as $\Delta \lambda_{L P} / \Delta n_{e x}$ we have the sensitivities, i.e D coefficients, of $-5.2 \mathrm{~nm} / 0.07$ and $-11.2 \mathrm{~nm} / 0.02$ for $\lambda_{L P 2}^{P 1}$ in these two ranges for the etched grating; the latter is more than seven times higher than the former.

Using the calibrating data in ref.7, we estimate that the LPG resonance shift induced by $1 \%$ sugar concentration change is $\sim 1 \mathrm{~nm} / \%$ in $1.42-1.44$ range for $\lambda_{L P 2}^{P 1}$ of the etched device. Thus, the sugar concentration change, as small as $0.1 \%$, can readily be detected by this grating using a standard interrogation system with an optical resolution of $\sim 0.1 \mathrm{~nm}$, and $0.01 \%$ change may be possible by using a tuneable laser with $0.01 \mathrm{~nm}$ resolution if the noise effect can be eliminated effectively. 

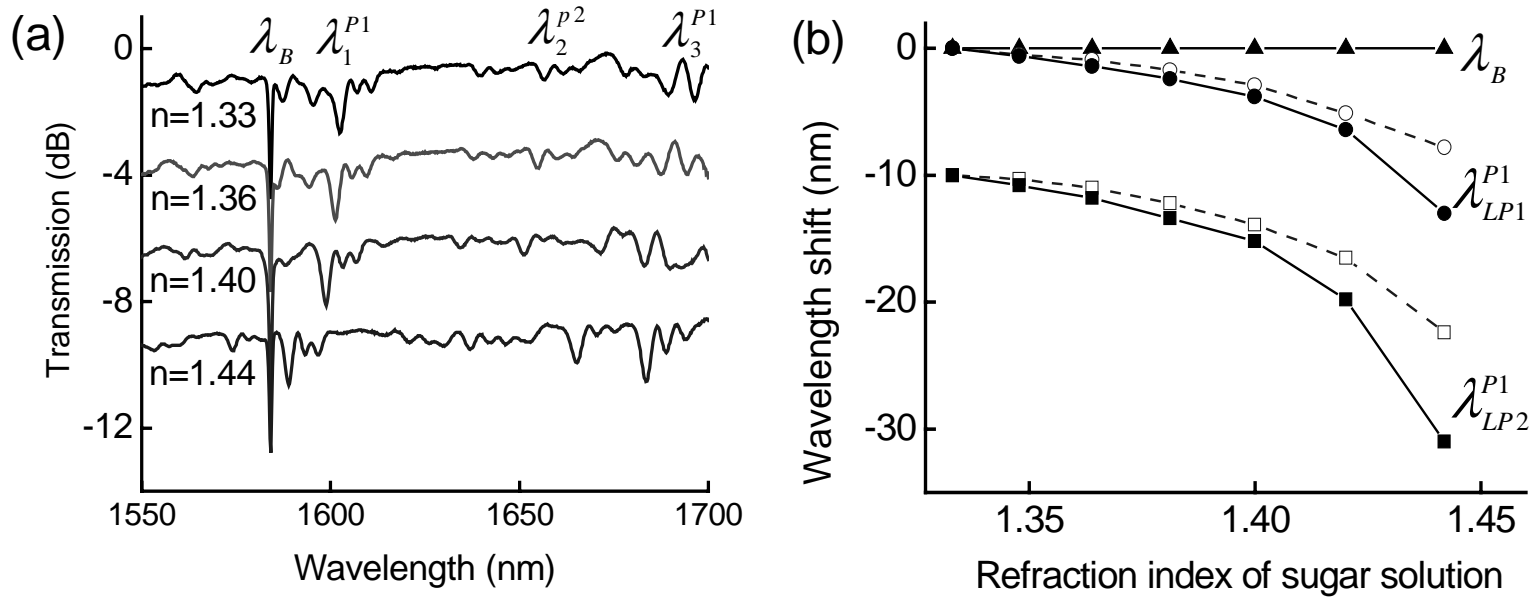

Fig.4 (a) Spectral evolution of the hybrid LPG/FBG in a series of sugar solutions; (b) Wavelength shifts of FBG resonance, two resonances of LPGs of unetched hybrid device (dashed curves) and etched hybrid devices (solid curves).

\section{RESULTS AND DISCUSSION}

The above calibrated temperature and ERI coefficients allow us to construct two sensitivity matrixes corresponding to the near-linear ERI ranges of 1.33-1.40, we have

$$
\left(\begin{array}{l}
\Delta T \\
\Delta n_{e x}
\end{array}\right)=\left[\begin{array}{ll}
11.3 p m /{ }^{o} C & 0 \\
48.1 p m /{ }^{o} C & -3.8 n m / 0.07
\end{array}\right]^{-1}\left(\begin{array}{l}
\Delta \lambda_{B} \\
\Delta \lambda_{L P 1}^{P 1}
\end{array}\right),
$$

the matrix will facilitate the decoupling between the temperature and ERI effects, achieving simultaneous measurement of temperature and ERI with high accuracy. The hybrid gratings may be used as optical chemsensors with advantages of high ERI sensitivity and temperature compensation,since the temperature information provided by the FBG response can be used as reference to extract accurate ERI encoded chemical concentration change information from LPG response. For the total ERI range (1.33-1.44), if we consider the nonlinear characteristics, the D coefficient in the sensitivity matrix may be expressed as polynomials of appropriate orders as functions of $\Delta n_{e x}$ :

$$
D=D_{0}+D_{1}\left(\Delta n_{e x}\right)+D_{2}\left(\Delta n_{e x}\right)^{2}+\ldots
$$

where the order of the polynomial is a function of the nonlinearity over the whole ERI range. The numerical method could be employed to decouple the signals, thus sensing the temperature and ERI simultaneously and accurately.

\section{CONCLUSIONS}


We have reported a hybrid LPG/FBG sensor device UV-written in D-fiber with significantly increased ERI and decreased temperature sensitivities by HF cladding etching. The temperature and ERI responses of the hybrid sensor have been carefully and comparatively characterized for etched and unetched devices. The etched device has been used to measure the concentrations of aqueous sugar solutions, demonstrating the potential capability for chemical, biochemical sensing.

\section{References:}

1. V. Bhatia and A. M. Vengsarkar, "Optical fiber long-period grating sensors", Opt. Lett. 21, 692-694 (1996).

2. L. Zhang, W. Zhang, and I. Bennion, "In-fiber grating optic sensors", in Fiber Optic Sensors, F. T. S. Yu and S. Yin eds., (Marcel Dekker, New York, 2002), pp. 123-181.

3. M. N. Ng, Z. H. Chen, and K. S. Chiang, "Temperature compensation of long-period fiber grating for refractiveindex sensing with bending effect", IEEE Photonics Technol. Lett. 14, 361-362 (2002).

4. X. W. Shu, B. A. L. Gwandu, Y. Liu, L. Zhang, and I. Bennion, "Sampled fiber Bragg grating for simultaneous refractive-index and temperature measurement", Opt. Lett. 26, 774-776 (2001).

5. H. J. Patrick, A. D. Kersey, and F. Bucholtz, "Analysis of the response of long period fiber gratings to external index of refraction", J. Lightwave Technol. 16, 1606-1611 (1998).

6. K. S. Chiang, Y. Liu, M. N. Ng, and X. Dong, "Analysis of etched long-period fiber grating and its response to external refractive index", Electron. Lett. 36, 966-967 (2000).

7. D. R. Lide, Eds., CRC Handbook of Chemistry and Physics, 79th edition, (CRC Press, Boca Raton, 1999). 